\title{
Molecular Cloning of Canine Thymus and Activation-Regulated Chemokine (TARC) Gene and Its Expression in Various Tissues
}

\author{
Sadatoshi MAEDA ${ }^{1)}$, Takuya MIZUNO ${ }^{1)}$, Kohei YAMASHITA ${ }^{2)}$, Keigo KURATA ${ }^{1)}$, Kenichi MASUDA $^{1)}$, \\ Koichi $\mathrm{OHNO}^{1)}$ and Hajime TSUJIMOTO ${ }^{1)}$ \\ ${ }^{1)}$ Department of Veterinary Internal Medicine, Graduate School of Agricultural and Life Sciences, The University of Tokyo, 1-1-1 Yayoi, \\ Bunkyo-ku, Tokyo 113-8657 and ${ }^{2)}$ Pharmaceutical Research Laboratory, Hitachi Chemical Co., Ltd., 4-13-1 Higashi-cho, Hitachi-shi, \\ Ibaraki 317-8555, Japan
}

(Received 22 February 2001/Accepted 22 May 2001)

ABSTRACT. Thymus and activation-regulated chemokine (TARC) is known as a functional ligand for CC chemokine receptor 4 (CCR4), which is selectively expressed on Th2 lymphocytes and induces selective migration of the cells to allergic lesions. In this study, we cloned canine TARC cDNA from canine thymus by RT-PCR with rapid amplification of cDNA ends (RACE) method. The canine TARC clone contained a full-length open reading frame encoding 99 amino acids and included four cysteine residues characteristic to CC chemokine family. The canine TARC cDNA showed $77.5 \%, 67.4 \%$, and $68.5 \%$ amino acid sequence similarity with human, mouse and rat homologues, respectively. Expression of TARC mRNA was detected not only in thymus but also in spleen, lymph node, lung and heart of the various normal dog tissues examined. TARC cDNA clone obtained in this study will be useful for further investigation on allergic diseases in dogs.

KEY WORDS: canine, cDNA cloning, thymus and activation-regulated chemokine.

J. Vet. Med.Sci. 63(9): 1035-1038, 2001

Allergic diseases such as atopic dermatitis, food allergy and allergic bronchitis are one of the most important problems in small animal practice. In human medicine, these allergic diseases were shown to be mediated by immune responses which involved in Th2-type lymphocytes producing cytokines such as IL-4, IL-5 and IL-13 [5, 9]. At the initiation of allergic diseases, the most noticeable finding is infiltration of inflammatory cells such as lymphocytes, eosinophils and mast cells into the lesion [6]. Of the lymphocytes, Th2 cells were shown to be dominant at the sites with allergic inflammation [7]. It has been shown that the migration of Th2 cells can be regulated by interaction with $\mathrm{CC}$ chemokines [1]. CC chemokine receptor 4 (CCR4) is selectively expressed on Th2 cells and its interaction with its ligand, thymus and activation-regulated chemokine (TARC), appears to play an important role for the migration of Th2 cells [2, 3]. It was reported that keratinocytes and bronchial epithelial cells were major cell sources to produce TARC protein in allergic legions [2, 8, 10]. Human [3], mouse [4], and rat (GenBank/EMBL/DDBJ accession number AF312687) TARC genes have been cloned so far. In the present study, we report the molecular cloning of canine TARC gene and its expression in various tissues to provide a fundamental information for the prospective studies on canine allergic diseases.

Total RNA was extracted from the thymus of a healthy dog kept for experimental purposes by use of the acid guanidine-phenol-chloroform method (RNAzol: Biotecx, Houston, TX). A cDNA sample was synthesized from $0.5 \mu \mathrm{g}$ of total RNA with a commercially available kit (RNA PCR Kit: PERKIN ELMER, Branchburg, NJ). Oligonucleotide primers to amplify a central region of canine TARC cDNA were designed based on the sequences conserved between human [3] and mouse [4] TARC cDNAs: forward primer, 5'-CACTTCAGATGCTGCTCCTGG-3' (nucleotide (nt) 36-56 in mouse TARC cDNA [4]) and reverse primer, 5' TGTTGGGGTCCGAACAGATG-3' (nt 284-265 in human TARC cDNA [3]). Using these primer pairs, canine TARC cDNA was amplified from the canine thymus cDNA with a commercial kit (RNA PCR Kit : PERKIN ELMER, Branchburg, NJ) according to the manufacturer's instructions. The PCR amplifications consisted of pre-denature $\left(95^{\circ} \mathrm{C}, 2 \mathrm{~min}\right)$ and 35 cycles of denaturation $\left(95^{\circ} \mathrm{C}, 1 \mathrm{~min}\right)$, annealing $\left(60^{\circ} \mathrm{C}, 1 \mathrm{~min}\right)$ and extension $\left(72^{\circ} \mathrm{C}, 7 \mathrm{~min}\right)$. The PCR generated a single DNA fragment of about $220 \mathrm{bp}$, and the product was cloned into a plasmid vector (pGEM-T easy vector: Promega, Madison, WI) followed by transformation of Escherichia coli competent cells (INV $\alpha F^{\prime}$ : Invitrogen, Carlsbad, CA). The PCR product cloned into plasmid was sequenced by dideoxy chain termination method (ABI Prism BigDye Primer Cycle Sequencing Ready Reaction Kit: Applied Biosystems, Foster City, CA). The clone obtained in this study was shown to have a high sequence similarity, $83.7 \%, 79.9 \%$ and $80.4 \%$, with human, rat, and mouse TARC cDNAs, respectively.

To amplify the 5' region of canine TARC cDNA, another primer pair was prepared: forward primer, 5'-TCCCCTCCTGGGCTCCTGGCACC-3' (nt 31-52 in human TARC cDNA [3]) and reverse primer, 5'-GGCAGCACTCCCGGCCCACG-3' (nt 103-84 in canine TARC cDNA obtained in this study). PCR amplification of the canine thymus cDNA with this primer pair generated a single DNA fragment of $134 \mathrm{bp}$, and its sequence was shown to be highly homologous to the 5' portion of human, rat, and mouse TARC cDNAs.

To amplify the 3' region of canine TARC cDNA, the 


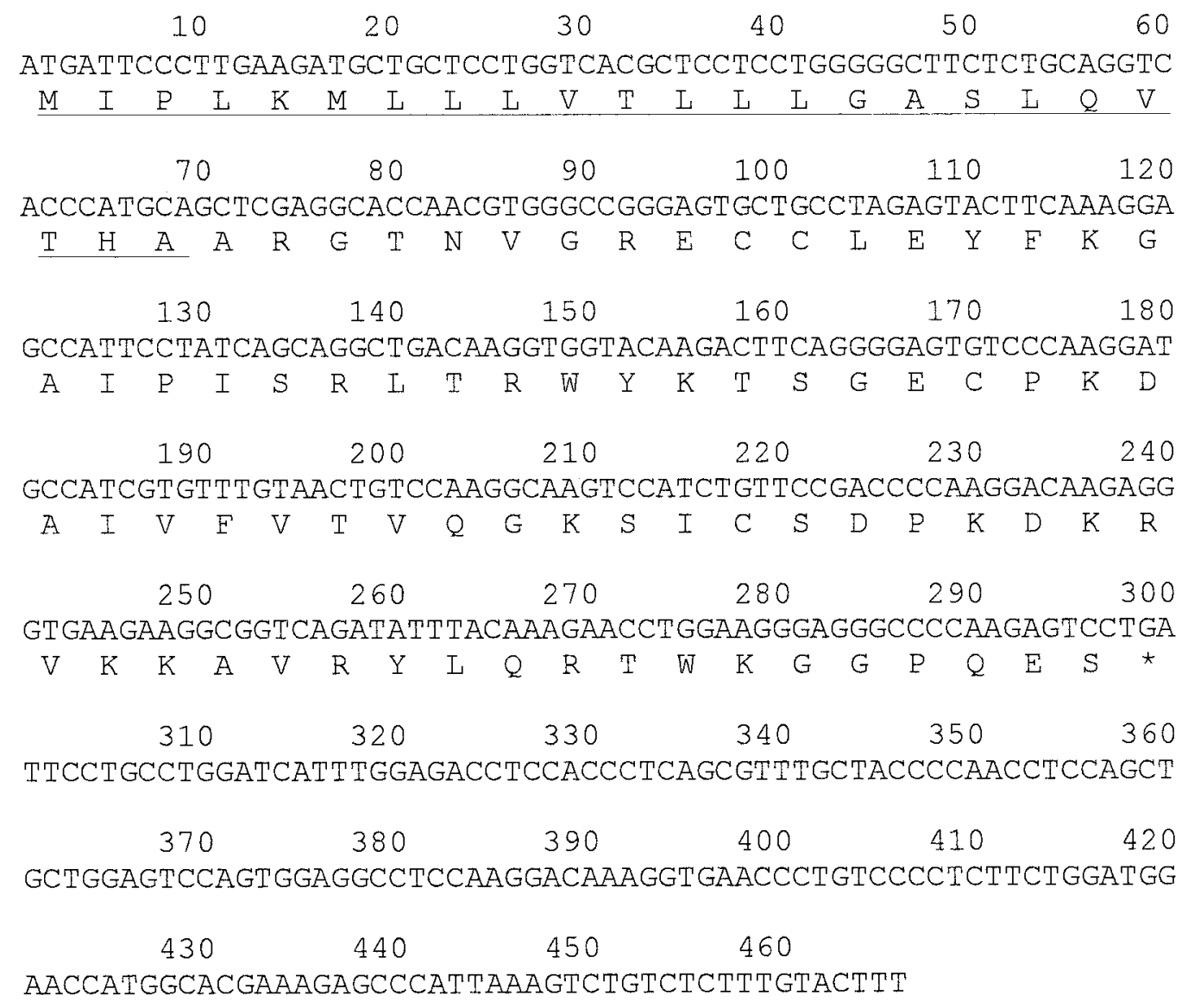

Fig. 1. Nucleotide and deduced amino acid sequences of canine TARC cDNA (GenBank/EMBL/DDBJ accession number AB054642). The numerals above each line refer to the nucleotide positions. The amino acid sequence with underline indicates the putative signal peptide. The asterisk after the amino acid sequence shows the position of the termination codon.

rapid amplification of cDNA ends (RACE) method was employed by using a commercially available kit (3' RACE System for Rapid Amplification of cDNA Ends: Life Technologies, Rockville, MD). Sequence of the forward primer was 5'-TAGAGTACTTCAAAGGAGCCAT-3' (nt 104124 in canine TARC cDNA obtained in this study) and that of the universal amplification primer was 5'-CUACUACUACUAGGCCACGCGTCGACTAGTAC-3' (3' RACE System for Rapid Amplification of cDNA Ends: Life Technologies). The PCR product was $341 \mathrm{bp}$ long and the sequence was shown to be highly homologous to those of human, rat and mouse TARC cDNAs.

By combining the sequences of the 5', central and 3' overlapping DNA fragments obtained in this study, a linear sequence corresponding to canine TARC cDNA was obtained (Fig. 1), which was 467 bp long and contained an entire open reading frame of canine TARC cDNA encoding 99 amino acid residues (GenBank/EMBL/DDBJ accession number AB054642). The amino acid sequence encoded by canine TARC cDNA was 5 or 6 residues longer than those encoded by human, mouse and rat homologues. The canine TARC cDNA cloned in this study was shown to have $77.5 \%, 67.4 \%$ and $68.5 \%$ amino acid sequence similarities with human [3], mouse [4] and rat TARC cDNAs, respectively (Fig. 2). In hydophilicity plot analysis, the canine TARC had a hydrophobic N-terminus corresponding the signal peptide identified in human, rat and mouse TARC cDNAs. Signal peptide cleavage site of canine TARC was assumed from its alignment with the homologues of other species, and the 24th amino acid from the initiation codon was tentatively assigned as being the first amino acid of the mature canine TARC (Fig. 2). The canine TARC cDNA was shown to have four cysteine residues including two adjacent cysteines characteristic to CC chemokine family.

We further examined the expression of TARC mRNA in normal dog tissues by RT-PCR. Total RNA samples were extracted from thymus, heart, lung, liver, spleen, kidney, adrenal gland, colon, small intestine, lymph node and skin of a healthy dog kept for experimental purposes. The cDNA samples of these tissues were prepared with the procedures 


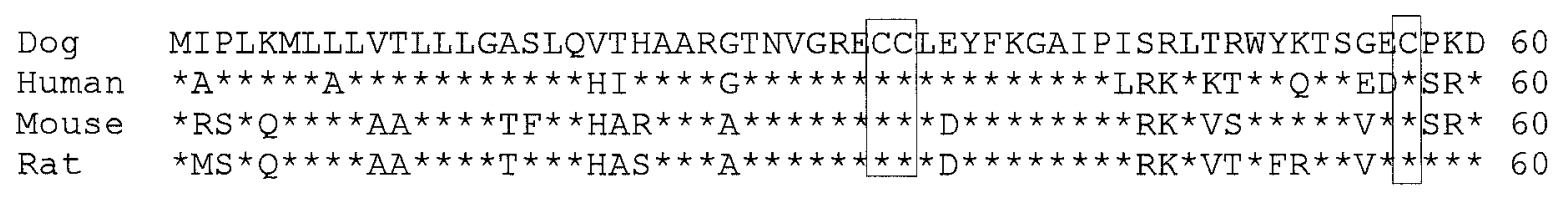

Dog AIVFVTVQGKSICSDPKDKRVKKAVRYLQRTWKGGPQES 99

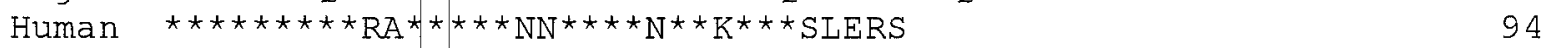

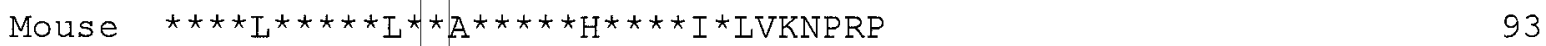

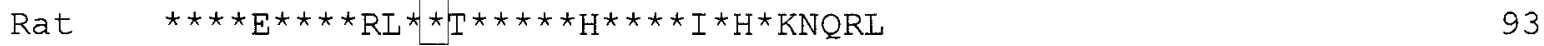

Fig. 2. Comparison of the deduced amino acid sequence of canine TARC cDNA with those of human, mouse and rat homologues. Asterisks indicate identities with amino acids of canine TARC sequence. Numerals on the right ends of each line show the total numbers of amino acid residues. Conserved cysteine residues are boxed.

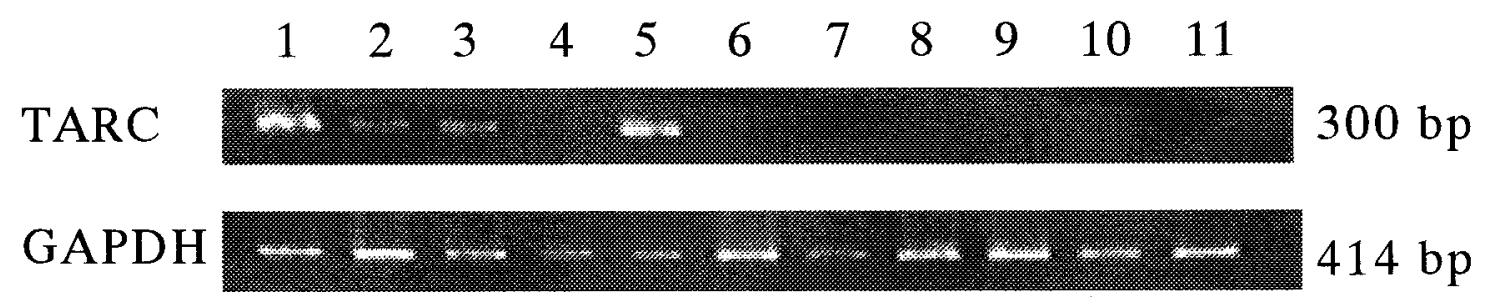

Fig. 3. Detection of canine TARC mRNA in various normal dog tissues. Canine TARC mRNA (upper lanes) and canine GAPDH mRNA (lower lanes) were detected by RT-PCR using primers specific to canine TARC and GAPDH cDNAs. Lane 1, thymus; lane 2, heart; lane 3, lung; lane 4, liver; lane 5, spleen; lane 6, kidney; lane 7, adrenal gland; lane 8, colon; lane 9, small intestine; lane 10, lymph node; lane 11, skin.

as described above. To amplify the full length of canine TARC cDNA, a primer pair was prepared: forward primer, 5'-ATGATTCCCTTGAAGATGCT-3' (nt 1-20 in canine TARC cDNA obtained in this study) and reverse primer, 5'TCAGGACTCTTGGGGCCCTC-3' (nt 300-281 in canine TARC cDNA). As an internal control, canine GAPDH cDNA was amplified, using a primer pair: forward primer, 5'-CTCATGACCACAGTCCATGC-3' (nt 514-533 in canine GAPDH cDNA, GenBank/EMBL/DDBJ accession number AB038240) and reverse primer, 5' - TGAGCTTGACAAAGTGGTCA-3' (nt 925-906 in canine GAPDH cDNA). Using the primer pair, PCR amplification was performed with the procedure as described above. The PCR products were electrophoresed through $3 \%$ agarose gel, and stained with ethidium bromide for visualization. Distinct bands of $300 \mathrm{bp}$ derived from canine TARC mRNA were detected in thymus and spleen, whereas relatively faint bands of the same size were found in heart, lung and lymph node (Fig. 3). Expression of TARC mRNA was not detected by RT-PCR in liver, kidney, adrenal gland and small intestine as well as in skin.

The nucleotide sequence reported in this study contained the entire open reading frame of canine TARC cDNA. The amino acid sequence encoded by canine TARC cDNA had four cysteine residues including two adjacent cysteines characteristic to $\mathrm{CC}$ chemokine family. In man, Northern blot analysis of TARC mRNA revealed that its high level expression in thymus and its low level expression in lung, colon and small intestine [3]. In this study, RT-PCR showed that the TARC mRNA was detected not only in thymus and lung but also in spleen, heart and lymph node. Unlike in man, expression of TARC mRNA was not detected in colon and small intestine in the dog in this study. It is conceivable that TARC mRNA is expressed in thymus and lung in general. But further study is required to know which tissue or organ expresses TARC in normal condition. Moreover, species difference of the expression of TARC in various tissues should be further investigated. TARC was recently reported to induce selective migration of Th2 cells and play an important role in the pathogenesis of allergic diseases such as atopic dermatitis [11] and bronchial asthma in humans [8]. Another study showed the increased level of TARC in the keratinocytes and epithelial cells at the allergic legions as well as the increased level of serum TARC protein in the patients with allergic diseases [8]. From these findings, it is highly plausible that production of TARC may also be increased in dogs with allergic diseases. Thus, TARC may be a novel clinical marker for the diagnosis and disease severity of allergic diseases in dogs. The present study will provide fundamental knowledge to understand the pathogenesis of allergic diseases in dogs. Furthermore, regulation of TARC production may be a novel therapeutic strategy for 
allergic diseases.

ACKNOWLEDGMENTS. This work was supported by the grants from the Ministry of Education, Science, Sports and Culture, and Special Coordination Funds for Promoting Science and Technology of the Science and Technology Agency of the Japanese Government. This work was also supported by a Grant-in-Aid of Recombinant Cytokine's Project provided by the Ministry of Agriculture, Forestry and Fisheries of Japan (RCP 1988-3110).

\section{REFERENCES}

1. Bleul, C. C. and Boehm, T. 2000. Eur. J. Immunol. 30: 33713379.

2. Imai, T., Nagira, M., Takagi, S., Kakizaki, M., Nishimura, M., Wang, J., Gray, P. W., Matsushima, K. and Yoshie, O. 1999. Int. Immunol. 11: 81-88.

3. Imai, T., Yoshida, T., Baba, M., Nishimura, M., Kakizaki, M. and Yoshie, O. 1996. J. Biol. Chem. 271: 21514-21521.

4. Lieberam, I. and Forster, I. 1999. Eur. J. Immunol. 29: 26842694.
5. Mazzarella, G., Bianco, A., Catena, E., De Palma, R. and Abbate, G. F. 2000. Allergy 55: 6-9.

6. Robinson, D. S., Hamid, Q., Ying, S., Tsicopoulos, A., Barkans, J., Bentley, A. M., Corrigan, C., Durham, S. R. and Kay, A. B. 1992. New. Engl. J. Med. 326: 298-304.

7. Romagnani, S., Parronchi, P., D’Elios, M. M., Romagnani, P., Annunziato, F., Piccinni, M. P., Manetti, R., Sampognaro, S., Mavilia, C., De Carli, M., Maggi, E. and Del Prete, G. F. 1997. Int. Arch. Allergy. Immunol. 113: 153-156.

8. Sekiya, T., Miyamasu, M., Imanishi, M., Yamada, H., Nakajima, T., Yamaguchi, M., Fujisawa, T., Pawankar, R., Sano, Y., Ohta, K., Ishii, A., Morita, Y., Yamamoto, K., Matsushima, K., Yoshie, O. and Hirai, K. 2000. J. Immunol. 165: 2205-2213.

9. Till, S., Durham, S., Dickason, R., Huston, D., Bungre, J., Walker, S., Robinson, D., Kay, A. B. and Corrigan, C. 1997. Immunology 91: 53-57.

10. Vestergaard, C., Bang, K., Gesser, B., Yoneyama, H., Matsushima, K. and Larsen, C. G. 2000. J. Invest. Dermatol. 115: 640-646.

11. Vestergaard, C., Yoneyama, H., Murai, M., Nakamura, K., Tamaki, K., Terashima, Y., Imai, T., Yoshie, O., Irimura, T., Mizutani, H. and Matsushima, K. 1999. J. Clin. Invest. 104: 1097-1105. 